\title{
Epidemiology, mechanisms and patterns of lower limb fractures At an accident service centre in Colombo, Sri Lanka.
}

\author{
Prabhashwara B, Akalanka N \\ National Hospital, Sri Lanka \\ Corresponding Author: \\ Buddhi Prabhashwara, \\ iD https://orcid.org/0000-0002-0567-9783
}

\begin{abstract}
:
Introduction: Lower limb fracture burden overwhelms most of the tertiary care facilities in Sri Lanka. Lack of enough data and a trauma registry in Sri Lanka has masked the associated epidemiology, patterns and the mechanisms of the lower limb fractures. Aim of our study was to analyse the data and put forward an initiative for further studies.
\end{abstract}

Methods: we collected data from the admissions with isolated lower limb fractures to the accident service Colombo South Teaching Hospital for a period of four months with the aid of an interviewer administered questionnaire.

Results: we had 323 admissions with lower limb fractures out of 4823 all the admissions. There were 334 fractures. Motor vehicle crashes were the commonest mechanism. Tibia/ fibula were the commonest bones to get fractured. Most of the culprits were in the age range of $21-40.68 \%$ of the males were under the influence of alcohol. $15 \%$ of the fractures were open.

Conclusion: we were able to emphasize the epidemiology, mechanisms and the fracture types as planned in the objectives.Key Words: Lower limb, fractures, epidemiology, patterns, mechanisms

\section{Introduction}

Lower limb fractures lead to a victim bedridden for a variable period of time and a significant morbidity and disability. There is a significant loss of productivity making it both a health and an economic hazard. Motor vehicle crashes, falls, sports injury and violence rarely spare lower limbs. Sri Lankan literature has not provided an enough space to it in comparison to the burden it causes. Absence of a well updated trauma registry data on the epidemiology, patterns, mechanisms and the severity of lower limb fractures are clearly affecting both research and therapeutic agenda. Purpose of this study is to analyze epidemiology, mechanisms and patterns of lower limb fractures in a tertiary care centre and thus put forward an initiative for future studies.

\section{Methodology}

This was a cross sectional study where all the individuals who were admitted to the accident service department of Colombo South Teaching Hospital (CSTH)Kalubowila with lower limb fractures for a period of four months were included.

The objectives were,

1. To assess the epidemiological data, mechanisms associated and the patterns of lower limb fractures

2. To assess correlation of epidemiology with the fracture patterns 
3. To assess correlation of fracture patterns and mechanisms

4. To assess the commonly fractured bones and sites

While all the patients admitted to the accident service with lower limb fractures during the study period were enrolled to the study following were excluded,

1. Patients with a history and radiologically visible old lower limb fractures

2. Patients with diagnosed metabolic and metastatic bone disease

3. Patients with benign or malignant bone tumors

4. Patients with concomitant upper extremity or pelvic fracture

5. Patients who died during the course of accident service admission

Interviewer administered questionnaire was used to collect data. Patients who were willing to participate were enrolled in accordance with inclusion and exclusion criteria and the questionnaire was administered. Data survey included demographic data, the mechanisms, and the associated fracture types. Certificate-of ethical clearance for the study was obtained from the Institutional ethical review committee CSTH, Kalubowila.

\section{Results}

Number of admissions during the study period of four months were 323. Number of all the lower limb fractures in the cohort were 334 as 11 patients had concomitant fractures. Most of the fractures were in the age group of 21-40,164/323(51\%); Table 1

Distribution of admissions according to age and gender

\begin{tabular}{|l|r|r|}
\hline \multicolumn{1}{|c|}{ Age } & Male & \multicolumn{2}{|c|}{ Female } \\
\hline $1-20$ & $8(73 \%)$ & $3(27 \%)$ \\
\hline $21-40$ & $104(63 \%)$ & $60(37 \%)$ \\
\hline $41-60$ & $70(79 \%)$ & $18(21 \%)$ \\
\hline $61-80$ & $13(26 \%)$ & $38(74 \%)$ \\
\hline$>81$ & $1(11 \%)$ & $8(89 \%)$ \\
\hline & $196(61 \%)$ & $127(39 \%)$ \\
\hline
\end{tabular}

Most of the fractures were due to motor vehicle crashes(MVCs); $60 \%$, followed by falls at home(FAH); 18\%, falls from height(FFH); $18 \%$ and sports injuries(SI); $4 \%$. Table 2

Table 2

Admission distribution according to age and the mechanisms

\begin{tabular}{|l|r|r|r|r|r|r|r|r|}
\hline \multirow{2}{*}{ Age in years } & \multicolumn{2}{|c|}{$\mathrm{MVC}(60 \%)$} & \multicolumn{2}{c|}{$\mathrm{FAH}(18 \%)$} & \multicolumn{2}{c|}{$\mathrm{FFH}(18 \%)$} & \multicolumn{2}{c|}{$\mathrm{SI}(4 \%)$} \\
\cline { 2 - 10 } & Male & Female & Male & Female & Male & Female & Male & Female \\
\hline $1-20$ & 6 & 3 & 0 & 0 & 0 & 0 & 2 & 0 \\
\hline $21-40$ & 85 & 39 & 0 & 3 & 11 & 16 & 8 & 2 \\
\hline $41-60$ & 48 & 6 & 2 & 8 & 20 & 4 & 0 & 0 \\
\hline $61-80$ & 6 & 2 & 3 & 34 & 4 & 2 & 0 & 0 \\
\hline$>81$ & 0 & 0 & 1 & 8 & 0 & 0 & 0 & 0 \\
\hline & $145(74)$ & $50(26)$ & $6(10)$ & $53(90)$ & $35(61)$ & $22(39)$ & $10(83)$ & $2(17)$ \\
\hline
\end{tabular}

Commonest fracture involved the tibia and fibula in our study (35\%), followed by metatarsophalangeal, neck of femur (NOF), ankle, patella, calcaneum and femur shaft. 
Table 3

Fracture types with age and gender

\begin{tabular}{|c|c|c|c|c|c|c|c|c|c|c|c|c|c|c|}
\hline \multirow{2}{*}{$\begin{array}{l}\text { Age in } \\
\text { years }\end{array}$} & \multicolumn{2}{|c|}{ NOF } & \multicolumn{2}{|c|}{ Femur } & \multicolumn{2}{|c|}{ Patella } & \multicolumn{2}{|c|}{ Tibia/fibula } & \multicolumn{2}{|c|}{ Ankle } & \multicolumn{2}{|c|}{ Calcaneum } & \multicolumn{2}{|c|}{ Meta/phal } \\
\hline & $M$ & $\mathrm{~F}$ & $M$ & $F$ & $M$ & $\mathrm{~F}$ & $M$ & $\mathrm{~F}$ & $\mathrm{M}$ & $\mathrm{F}$ & $M$ & $\mathrm{~F}$ & $M$ & $F$ \\
\hline $1-20$ & 0 & 0 & 2 & 0 & 0 & 1 & 4 & 2 & 2 & 0 & 0 & 0 & 1 & 0 \\
\hline $21-40$ & 0 & 0 & 5 & 2 & 10 & 6 & 40 & 26 & 15 & 8 & $\mathrm{O}$ & 0 & 34 & 18 \\
\hline $41-60$ & 0 & 2 & 4 & 1 & 4 & 2 & 28 & 8 & 8 & 4 & 16 & 0 & 14 & 4 \\
\hline $61-80$ & 3 & 35 & 0 & 0 & 1 & 0 & 5 & 2 & 1 & 1 & 2 & 0 & 2 & 0 \\
\hline$>81$ & 1 & 8 & 0 & 0 & 0 & 0 & 0 & 0 & 0 & 0 & 0 & 0 & 0 & 0 \\
\hline & 4 & 45 & 11 & 3 & 15 & 9 & 77 & 38 & 26 & 13 & 18 & 0 & 51 & 22 \\
\hline
\end{tabular}

While none of the female admissions were under the influence of alcohol and 133/196(68\%)males were under the influence.

Table 4

Age and the number of males under the influence of alcohol

\begin{tabular}{|l|r|r|}
\hline \multicolumn{1}{|c|}{ Age in years } & \multicolumn{2}{|c|}{ Male } \\
\hline $1-20$ & 8 & $3 / 8$ \\
\hline $21-40$ & 104 & $82 / 104$ \\
\hline $41-60$ & 70 & $48 / 70$ \\
\hline $61-80$ & 13 & $0 / 13$ \\
\hline$>81$ & 1 & 0 \\
\hline & 196 & 133 \\
\hline
\end{tabular}

$49 / 334(15 \%)$ of the fractures were open. Most of the open fractures were metatarsophalangeal fractures, $20 / 49$.

Table 5

Mechanisms, fracture types and nature

\begin{tabular}{|l|l|l|l|l|l|l|l|l|}
\hline \multirow{2}{*}{ Fracture type } & MVC & \multicolumn{2}{l|}{ FAH } & \multicolumn{2}{l|}{ FFH } & \multicolumn{2}{l|}{ SI } \\
\cline { 2 - 10 } & Close & Open & Close & Open & Close & Open & Close & Open \\
\hline NOF & 0 & 0 & 45 & 0 & 4 & 0 & 0 & 0 \\
\hline Femur & 10 & 2 & 0 & 0 & 2 & 0 & 0 & 0 \\
\hline Patella & 19 & 3 & 0 & 0 & 0 & 0 & 2 & 0 \\
\hline Tibia/fibula & 76 & 16 & 0 & 0 & 17 & 3 & 3 & 0 \\
\hline Ankle & 6 & 2 & 14 & 0 & 12 & 0 & 5 & 0 \\
\hline Calcaneum & 0 & 0 & 0 & 0 & 15 & 3 & 0 & 0 \\
\hline Meta/phal & 48 & 20 & 0 & 0 & 5 & 0 & 2 & 0 \\
\hline & 159 & 43 & 59 & 0 & 55 & 6 & 12 & 0 \\
\hline
\end{tabular}

\section{Discussion}

Lower limb fracture burden overwhelms most of the tertiary care facilities in SriLanka. It is a reality throughout the world as well. Lower limb fractures account for $1 / 3$ of all the fractures. Epidemiological data regarding them is minute in Sri Lankan as well as in world literature. In our data collection for the period of four months total number of admissions with 'isolated' lower limb fractures were 323/4823(6.7\%). As individuals with concomitant upper extremity and pelvic fractures were excluded from the study true lower limb fracture frequency among accident service admissions is more. According to J A Kaye and colleagues, in United Kingdom the incidence of lower 
limb fractures for 1000 person years is 2.9 for males and 3.1 for females ${ }^{1}$. For the analysis of true incidence large population based study is required.When considering the poor road traffic safety and the numerous household and occupational hazards our rates should and will be far ahead of the UK rates. We had $127 / 323(39 \%)$ female admissions and $196 / 323(61 \%)$ male admissions. A study in a tertiary care unit in Ahmedabad by Goel SA and colleagues $75 \%$ of the lower limb fractures were in males $^{2}$. In a high volume orthopaedic centre in UP India $61 \%$ of the lower limb fractures were in males $^{3}$. Our statistics are quiet similar to UP India study. Differences observed with the Ahmedabad study may be due to cultural and ethnic variables in the populations considered. Percentage of female lower limb fractures due to MVCs was $26 \%$ in our study. In a study by Weerawardena WAK and colleagues all the female admissions following Road traffic accidents(RTAs) to General HospitalPolonnaruw $26 \%{ }^{4}$. In a study in Tamil Nadu $27 \%$ of all the admissions to an orthopedic trauma centre were females ${ }^{5}$. As we have considered only the lower limb fractures even after leaving road traffic culture differences in two areas considered behind percentage of $26 \%$ is noteworthy. More female motorcyclists in roads with poor safety precautions is and will be a health hazard in near future. Non of the female victims were under the influence of alcohol. Though $133 / 196(68 \%)$ male victims were under the influence. All who were under the influence of alcohol were either in MVCs category or FFHs category. 3/6(50\%), 73/85(86\%), 35/48(72\%) male MVCs victims in age ranges from 1-20, 21-40 and 41-60 respectively. These figures are significant as the intoxication must have been the most influencing variable which led to the lower limb fracture. As all most all male FFHs were work place injuries fact of $9 / 11$ and $13 / 20$ culprits in age ranges of 21-40 and 41-60 respectively were under the influence at the time of the event is a major consideration to the relevant authorities and the construction companies regarding the work place safety.

The age range with highest lower limb fracture frequency among both males and females in our study was 21-40. MVCs being the commonest mechanism for both genders in the same age range demonstrates the risk taking behaviors of the youth which needs to be addressed properly. Almost $90 \%$ of the male lower limb fractures were clustered within the age range of 21-60(years). In females there was a bimodal distribution of the fractures. While 60/127 were in the age range of 21$40,38 / 127$ were in the range of 61-80. Commonest mechanism of fractures among female in age range of 61-80 was FAH and the commonest fracture was Neck of femur. Ahmedabad study too demonstrates the bimodal distribution of fractures with the age.MVCs were the commonest mechanism in our study 195/323(60\%). Ahmedabad study it accounted for $72 \%$ of the lower limb fractures. That study is similar in nature to our study as it too has been carried out in an emergency department with casuality admissions. In UP India study MVCs caused $30 \%$ of the lower limb fractures. It has mainly focused on osteoporotic fractures and has been conducted in an orthopedic unit. In a study in Iran 70\% of all the extremity fractures in MVCs were in lower extremities ${ }^{6}$. An article published in Singapore medical journal states $58 \%$ of motorcyclists sustained lower limb fractures during crashes ${ }^{7}$. As studies in our nature are limited in literature comparative percentages are difficult to find out. Literature regarding the Incidence of lower limb fractures associated with falls and sports injury further scarce than MVCs. Same Ahamedabad study shows falls at home, falls from height and sports injury account for $16 \%, 6 \%$ and $2 \%$ of the lower limb injuries in an accident service respectively. In our study those percentages were 18, 18 and 4 respectively. High percentage of lower limb fractures with FFHs is a fact to be considered as the work place injuries can be avoided with caution and precautions. Commonest fractures in our study was tibia/fibula shaft and the tibial platue which together constituted $35 \%$ followed by metatarso phalangeal fractures(22\%). Neck of femur fractures together with femur shaft fractures were more or less similar in percentage to metatarso phalangeal fractures. In Singapore study fibular fractures had the highest frequency while femoral fractures had the lowest, being $44 \%$ and $7 \%$ respectively. In the Iranian study out of all the extremity fractures among MVC victims $37 \%$ were tibio fibular, which was highest in frequency. Femur, foot and the patella had $14 \%, 5 \%$ and $2 \%$ of fracture frequencies respectively. In the Ahamedabad study femur was the commonest bone to get fractured, followed by tibia. In the UK 
study of epidemiology of lower limb fractures in general practice ankle fractures were the commonest followed up by tibia fibula, tarso metatarsal and phalanges. Thesedifferences in fracture distribution can be attributed to the nature of the MVCs in our setup. In most of our MVCs the culprit vehicle was a motor cycle or a three wheeler. And the collision was either between the two vehicles or one slipping or toppling on itself. So the energy was not high enough in most of the instances to fracture a femur. Low level impacts and motor cycle foot rest collisions are responsible for the high frequency of metatarso phalangeal fractures observed. Most of these metatarso phalangeal fractures were compound and communuited and ultimately led the way to deformities, osteomyelitis and foremost out of work for significant period of time. While femur and tibia fractures are getting the priority of the orthopedic team metatarso phalangeal fractures are ill treated in most of the instances. As protective head gears are being introduced frequently to the market the foot protective gears also need to be manufactured. When the mechanisms and the fracture types were considered separately calcaneal fractures and ankle fractures taken together surpassed the number of tibia and fibula fractures in FFHs.

In the above mention Singapore study out of $58 \%$ of the lower limb fractures among motorcyclists $84 \%$ were closed fractures, while $13 \%$ were open. Though our study is different to the Singapore study, percentage of the open fractures was more or less similar, 49/334(15\%). Almost third of the metatarso phalangeal fractures were open $20 / 68(30 \%)$. It is a fact to be considered seriously as we have noted out previously. Incidence of lower limb fractures in sports injury has not been analyzed in literature adequately. In our study only $12 / 334(4 \%)$ fractures were due to SIs. Isolated lower limb injury frequencies has been analyzed in the west in sports like netball and ice hockey. As sports culture in Sri Lanka is significantly different, the specific data of bigger magnitude has to be collected to come to a conclusion about the patterns in this group ${ }^{8}$.

\section{Conclusion}

'Isolated lower limb fractures contribute to over $7 \%$ of all the admissions to an accident service in our cohort. This is an alarming proportion and an eye opener to the authorities. High frequency of female lower limb fractures and the associated MVCs is a matter of concern. Alcohol being the major modifiable variable in male lower limb fractures associated with both MVCs and FFHs indicates effective preventive measures could be applied. 'The age range with the highest lower limb fracture frequency is 21-40 years, our data have re emphasized the risk taking behavior of the youth just like most of the other studies have done earlier. High number of compound metatarsophalangeal fractures and the morbidity associated with them is a matter to be analyzed and discussed further in order to apply preventive and protective measures.

\section{References}

1. Kaye JA, H Jick H. Epidemiology of lower limb fractures in general practice in the United Kingdom. Injury Prevention 2004;10:368-374. DOI: http://dx.doi.org/10.1136/ip.2004.005843

2. 2.Goel SA, Bhavsar NM , Makwana H , Lil NA , Patel PR. Epidemiology and patterns of lower limb injuries at a tertiary care hospital in Ahmedabad.International Journal of Medical Research and Review, June, 2015/ Vol 3/Issue 5.DOI: 10.17511/ijmrr.2015.i5.094

3. Rajneesh Jindal, Neera Jindal, AnkurDass. A retrospective study of prevalence of lower limb fractures. Journal of Advanced Medical and Dental Sciences Research |Vol. 4|Issue 6|November - December 2016 23. DOI: 10.21276/jamdsr.2016.4.6.7

4. W. A. K. Weerawardena, T. G. L. H. M. Thennegadara, H. G. V. Priyantha, P. A. C. G. S. Chandrasiri, W. S. I. Disanayaka. Road traffic accidents in Polonnaruwa District: Analysis of patients admitted to a surgical unit. Journal of the Ruhunu Clinical Society, 23(1), pp.25-29. DOI: http://doi.org/10.4038/jrcs.v23i1.46 
5. A.Manoharan, P. Selvaraj, P. Vasanthamani. Epidemiology of Orthopedic Injuries among Patients Attending a Major Trauma Centre in Tamilnadu. National Journal of Research in Community Medicine. Vol.5. Issue 2. Apr.-Jun.-2016(126-131)

6. Mehrdad Mahdian, Mohammad R. Fazel, MojtabaSehat, GholamrezaKhosravi. Epidemiological Profile of Extremity Fractures and Dislocations in Road Traffic Accidents in Kashan, Iran:a Glance at the Related Disabilities. The Archive of Bone and Joint Surgery, (5)3 Page 186-192

7. KTham, E Seow, G Lau. Patterns of injuries in helmeted motorcyclists in Singapore. Emerg Med J. 2004 Jul; 21(4): 478-482. DOI: 10.1136/emj.2002.002261

8. Finch C, Da Costa A, Stevenson M, Hamer P, Elliott B.Sports injury experiences from the Western Australian sports injury cohort study. Aust N Z J Public Health. 2002 Oct;26(5):462-7. DOI: https://doi.org/10.1111/j.1467-842X.2002.tb00348.x 